DAMTP-1999-67

hep-th/9905156

\title{
Supersymmetry and Generalized Calibrations
}

\author{
J. Gutowski, G. Papadopoulos and P.K. Townsend \\ DAMTP, Univ. of Cambridge, Silver St., \\ Cambridge CB3 9EW, UK
}

\begin{abstract}
A static minimal energy configuration of a super p-brane in a supersymmetric $(n+1)$-dimensional spacetime is shown to be a 'generalized calibrated' submanifold. Calibrations in $\mathbb{E}^{(1, n)}$ and $A d S_{n+1}$ are special cases. We present several M-brane examples.
\end{abstract}

\section{INTRODUCTION}

A super p-brane [1] 3] is a charged p-dimensional object moving in superspace, with a charge equal to its tension, which we set to unity. If the superspace is a supersymmetric one then the $(\mathrm{n}+1)$-dimensional spacetime will admit a timelike Killing vector field $k$ and the motion will conserve a corresponding energy $E$. The fact that the p-brane is charged means that it couples to a superspace $(\mathrm{p}+1)$-form potential $A$. There are therefore two contributions to the energy density of a static brane, one proportional to the p-volume density, the other an 'electrostatic' energy density. The p-volume density is $\sqrt{\operatorname{det} m}$ where $m$ is the induced worldspace p-metric. The electrostatic energy density is the worldspace dual of the p-form induced by $i_{k} A$; let us call this $\Phi$. Thus, as we shall confirm below, stable static bosonic solutions to the worldvolume field equations (the 'branewave' equations) minimize a potential energy functional of the form

$$
E=\int d^{p} \sigma[\nu \sqrt{\operatorname{det} m}+\Phi],
$$

where $\sigma^{i}(i=1, \ldots, p)$ are the worldspace coordinates, and $\nu$ is a redshift factor; for static spacetimes $\nu=$ $\sqrt{-k^{2}}$. We shall call these static configurations 'minimal worldspaces'. For a vacuum superspace background the spacetime is $(\mathrm{n}+1)$-dimensional Minkowski space, $\mathbb{E}^{(1, n)}$. In this case $\Phi=0$ because the (p+1)-form $A$ is purely fermionic. In addition, $\nu=1$, so in this case a minimal worldspace is a minimal surface in $\mathbb{E}^{n}$ in the usual sense. We see that super p-branes provide a natural framework for an extension of the notion of a minimal surface in $\mathbb{E}^{n}$ to minimal worldspaces in more general Riemannian spaces.

Supersymmetry did not play a major role in the above discussion and it might be deemed an unnecessary complication. However, a large class of minimal surfaces in $\mathbb{E}^{n}$ are calibrated surfaces for which the calibrating p-

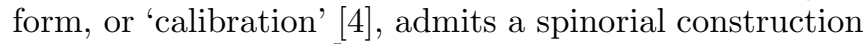
[5]. Let $x^{m}=\left(x^{0}, x^{I}\right)$ be the Minkowski spacetime coordinates, with $x^{I}(I=1, \ldots, n)$ being cartesian coordinates on $\mathbb{E}^{n}$. Then, for $p=1,2 \bmod 4$, the $\mathrm{p}$-form calibration takes the form

$$
\varphi=d x^{I_{1}} \wedge \ldots \wedge d x^{I_{p}} \epsilon^{T} \Gamma_{0 I_{1} \ldots I_{p}} \epsilon
$$

where $\epsilon$ is a constant real spinor 7 normalized so that

$$
\epsilon^{T} \epsilon=1,
$$

and $\Gamma_{m_{1} \ldots m_{k}}$ are antisymmetrized products of the Minkowski spacetime Dirac matrices. Many calibrations of this form have been shown [6] to calibrate supersymmetric intersecting brane configurations in M-theory (for which $\epsilon$ is a real 32-component spinor of the 11dimensional Lorentz group). Similar calibrations were earlier discussed in the context of Calabi-Yau compactifications of string theory [7]. The essential point is that, given a tangent p-plane $\xi$, we can write $\left.\varphi\right|_{\xi}$ as

$$
\left.\varphi\right|_{\xi}=\sqrt{\operatorname{det} m} \epsilon^{T} \Gamma_{\xi} \epsilon
$$

where $\Gamma_{\xi}$ is the matrix

$$
\Gamma=\frac{1}{p ! \sqrt{\operatorname{det} m}} \varepsilon^{i_{1} \ldots i_{p}} \partial_{i_{1}} x^{I_{1}} \ldots \partial_{i_{p}} x^{I_{p}} \Gamma_{0 I_{1} \ldots I_{p}},
$$

evaluated at the point to which $\xi$ is tangent. Given the above restriction on the values of $p$, we have

$$
\Gamma^{2}=1 \text {. }
$$

It follows that $\left.\varphi\right|_{\xi} \leq \operatorname{vol}_{\xi}$ for all $\xi$. Since $\varphi$ is also closed this means that it is a calibration. The 'contact set' of the calibration is the set of p-planes for which this inequality is saturated. By the theorem of Harvey and Lawson, these are the tangent planes of a minimal surface [4].

We see from (4) that an alternative characterization of the contact set is that set of p-planes $\xi$ for which

$$
\Gamma_{\xi} \epsilon=\epsilon .
$$

Because of (6) this equation is automatically satisfied for any given tangent p-plane $\xi$ and, since $\operatorname{tr} \Gamma=0$, the solutions $\epsilon$ belong to a ' $1 / 2$ supersymmetric' subspace

\footnotetext{
${ }^{*}$ The requirement that $\epsilon$ be real restricts the spacetime dimension, and hence the space dimension $n$; the extent to which the constraints on $p$ and $n$ may be relaxed will be discussed below.
} 
of spinor space. However, this solution space generally varies as $\xi$ varies over the contact set so the solution space of the set is generally smaller. The connection of calibrations to supersymmetry observed in [6] is based on the fact that the matrix $\Gamma$ is closely related to a matrix with similar properties appearing in the $\kappa$-symmetry transformations of the worldvolume fields of the super p-brane in its Lagrangian formulation. In fact, for time-independent worldvolume fields in the gauge $x^{0}=t$ the latter matrix reduces to the one of (5), but in this context the condition (7) is just the condition for preservation of some fraction of supersymmetry; the total number of preserved supersymmetries is the dimension of the solution space for $\epsilon$. Note that although the specific form of the calibrating p-form given above required restrictions on both $p$ and $n$, the results can be extended without difficulty to all those pairs $(p, n)$ for which there exists a super p-brane action. The possibilities were classified in [3] under the assumption that the only bosonic worldvolume fields are scalars, and these are the cases that we have in mind here. The 11-dimensional supermembrane [2] is a simple example with $(p, n)=(2,10)$. Of course, many of the results to be obtained will apply to other branes such as D-branes and the M5-brane if one sets to zero any nonscalar worldvolume fields. In fact, most discussions to date of the relevance of calibrations to branes have been in the context of the M5-brane.

Most previous discussions have also concentrated on the case of branes in a flat Minkowski background. In considering the extension to non-flat backgrounds it is convenient to distinguish between those for which the energy functional of a p-brane is still equal to the integral of the p-volume and those for which the more general formula (1) is required. In the former case, a static p-brane is again a minimal surface and supersymmetric minimal p-surfaces are calibrated by p-form calibrations in much the same way as in the above discussion. The examples of [7] are in this category. As we shall show here, the recently discussed [8] 'lump' solitons on the M2-brane in an M-monopole background provide further examples of Kähler calibrations of this type, and there are a number of related Kähler calibrations that we discuss. If, on the other hand, a static brane of minimal energy is not a minimal surface then it cannot be a calibrated surface in the sense of 洵, but it may still be a calibrated surface in a more general sense because it may be calibrated by a form that is not closed; examples are provided by the 'adS' calibrations [9] which calibrate p-surfaces of minimum p-brane energy in anti-de Sitter backgrounds. The adS examples suggest a definition of a 'generalized calibration', which we present here; we then show, by a straightforward extension of an argument in [9], that p-surfaces calibrated by such generalized calibrations are 'minimal worldspaces'. We discuss a number of examples provided by M-branes in 1/2-supersymmetric M-theory backgrounds.

Another purpose of this article is to show how the connection between calibrations and super p-branes fol- lows directly from the supersymmetry algebra of Noether charges [10,11. In particular, we shall see that all the properties of generalized calibrations arise naturally in this context. For this reason we shall begin with this analysis. Since the supersymmetry charges are integrals of functions on phase space, it is natural to start from the Hamiltonian formulation, the details of which can be found in 12 14. Having defined the notion of a generalized calibration and proved the associated minimal worldspace property of calibrated surfaces, we then move on to the explicit examples.

\section{THE SUPERSYMMETRY ALGEBRA AND THE CALIBRATION BOUND}

We begin by making precise the constructions alluded to in the opening paragraph. Let $Z^{M}=\left(x^{m}, \theta^{\mu}\right)$ be the superspace coordinates. The geometry of superspace will be assumed to be determined by the Lorentz frame 1-forms $E^{A}=\left(E^{a}, E^{\alpha}\right)$ and the $(\mathrm{p}+1)$-form potential $A$; we will assume throughout that the only non-vanishing component fields are bosonic. The superspace $(\mathrm{p}+1)$ form $A$ appears in the Lagrangian form of the action via its pullback to the worldvolume and the integrand can be written in the form $\dot{Z}^{M} \mathcal{A}_{M}$ where the dot denotes differentiation with respect to an arbitrary worldvolume time $t$; the coefficients $\mathcal{A}_{M}$ are the components of the oneform $\mathcal{A}$ obtained by contraction of $A$ with the $p$ tangent vectors spanning a tangent p-plane $\xi$ to the p-dimensional worldspace, i.e. $\mathcal{A}=\left.A\right|_{\xi}$. Now let $x^{m}=\left(x^{0}, x^{I}\right)$, where $k=\partial / \partial x^{0}$ is the timelike Killing vector field, and fix the time reparameterization invariance by choosing $x^{0}=t$. Then

$$
\dot{Z}^{M} \mathcal{A}_{M}=\Phi+\dot{x}^{I} \mathcal{A}_{I}+\dot{\theta}^{\mu} \mathcal{A}_{\mu}
$$

where $\Phi$ is a superfield with the electrostatic potential density as its lowest component. As is customary, we use the same symbol to denote a superfield and its lowest component. Note that $\Phi$ is the worldspace dual of the pullback of $i_{k} A$. The worldspace metric is $m_{i j}=E_{i}^{a} E_{j}^{b} \eta_{a b}$ where $E_{i}{ }^{a}=\partial_{i} Z^{M} E_{M}{ }^{a}$ and $\eta$ is the Minkowski metric. Omitting fermions it reduces, in the $x^{0}=t$ gauge, to

$$
m_{i j}=\partial_{i} x^{I} \partial_{j} x^{J} g_{I J}
$$

where $g_{I J}$ is the metric on a spatial section of spacetime, i.e. the p-metric on worldspace is induced from the nmetric on space.

The super p-brane Lagrangian density can be written as

$$
\mathcal{L}=\dot{Z}^{M} P_{M}-s^{i} E_{i}{ }^{a} \tilde{p}_{a}-\frac{1}{2} \lambda\left(\tilde{p}^{2}+\operatorname{det} m\right)
$$

where $s^{i}$ and $\lambda$ are Lagrange multiplers, and

$$
\tilde{p}_{a}=E_{a}{ }^{M}\left(P_{M}+\mathcal{A}_{M}\right) .
$$


This is not its fully canonical form because $\kappa$-symmetry implies a constraint for which there is as yet no corresponding Lagrangian multiplier. However, this constraint affects only the fermionic variables. If we set all worldvolume fermions to zero and use the identity $\partial_{i} x^{m} \mathcal{A}_{m} \equiv 0$ then

$$
\mathcal{L}=\dot{x}^{m} p_{m}-s^{i} \partial_{i} x^{m} p_{m}-\frac{1}{2} \lambda\left(\tilde{p}^{2}+\operatorname{det} m\right)
$$

where, now, $\tilde{p}^{2}=g^{m n} \tilde{p}_{m} \tilde{p}_{m}$, with $\tilde{p}_{m}=p_{m}+\mathcal{A}_{m}$ and $g^{m n}$ the inverse of the spacetime metric $g_{m n}=$ $E_{m}{ }^{a} E_{n}{ }^{b} \eta_{a b}$. Now set

$$
p_{m}=\left(-\mathcal{H}, P_{I}\right)
$$

and impose the $x^{0}=t$ gauge, to get

$$
\mathcal{L}=\dot{x}^{I} p_{I}-\mathcal{H}-s^{i} \partial_{i} x^{I} p_{I}
$$

where the Hamiltonian density $\mathcal{H}$ is found by solving the Hamiltonian constraint $\tilde{p}^{2}+\operatorname{det} m=0$. This yields

$$
\mathcal{H}=\Phi+v^{I} \tilde{p}_{I}+\nu\left[g_{(n)}^{I J} \tilde{p}_{I} \tilde{p}_{J}+\operatorname{det} m\right]^{1 / 2}
$$

where

$$
v^{I}=\frac{g^{t I}}{\left(g^{t t}\right)}, \quad \nu=1 / \sqrt{-g^{t t}}
$$

and $g_{(n)}^{I J}$ is the inverse of the spatial n-metric $g_{I J}$. For simplicity we now restrict to static spacetimes, for which $v^{I}$ vanishes. In this case $\nu=\sqrt{-k^{2}}$. For static worldvolume configurations we may set $\tilde{p}_{I}=0$ and the Hamiltonian density reduces to

$$
\mathcal{H}=\Phi+\nu \sqrt{\operatorname{det} m} .
$$

Because $k=\partial_{t}$ is Killing, $m_{i j}$ and $\Phi$ are timeindependent. The integral of $\mathcal{H}$ over the worldspace yields the potential energy functional $E$ of (11).

Supersymmetries of the background are associated with Killing spinor fields. In many simple cases these take the form of a function times a constant spinor $\epsilon$ subject to a constraint of the form $\Gamma_{*} \epsilon=\epsilon$ where the constant matrix $\Gamma_{*}$ is characteristic of the background. In particular, all 1/2-supersymmetric backgrounds have Killing spinors of this form with $\Gamma_{*}$ a traceless matrix such that $\Gamma_{*}^{2}=1$. We shall assume here that the background superspace is of this type. In addition, we continue to make the inessential, but simplifying, assumptions that $p=1,2$ $\bmod 4$, and that spinors are real. In these cases we may take the charge conjugation matrix to equal $\Gamma^{\underline{0}}$, where the underlining indicates a constant Lorentz frame matrix. The matrix $\Gamma$ of (5) can be generalized to non-flat spacetimes by taking

$$
\sqrt{\operatorname{det} m} \Gamma=\Gamma_{\underline{0}} \gamma
$$

where

$$
\gamma=\frac{1}{p !} \varepsilon^{i_{1} \ldots i_{p}} \partial_{i_{1}} x^{I_{1}} \cdots \partial_{i_{p}} x^{I_{p}} \Gamma_{I_{1} \ldots I_{p}}
$$

Note that, given the restriction on the values of $p$, we have

$$
\gamma^{2}=-(-1)^{p} \operatorname{det} m
$$

Given our assumptions about the background the supersymmetry charges will take the form of spinor functionals $Q_{\alpha}$ subject to the constraint $Q \Gamma_{*}=Q$. We will proceed by taking this projection to be implicit, and checking consistency at the end. With this understood we can write the supersymmetry anticommutator as

$$
\{Q, Q\}=\Gamma^{\underline{0}} \int d^{p} \sigma \nu\left[\Gamma^{a} \tilde{p}_{a}+\gamma\right] .
$$

This result has not been directly established in full generality but it agrees with the flat space case 10 and those non-flat cases that have been analyzed [11]. That it must be correct follows indirectly from considerations of $\kappa$-symmetry, as will now be explained. The $\kappa$-symmetry transformations were given in the required form in [13. In particular, the transformations of the fields $Z^{M}$ are such that $\delta_{\kappa} Z^{M} E_{M}{ }^{a}=0$ and

$$
\delta_{\kappa} Z^{M} E_{M}^{\alpha}=\left[\Gamma^{a} \tilde{p}_{a}-(-1)^{p} \gamma\right] \kappa
$$

Since $E_{m}{ }^{\alpha}$ vanishes for a bosonic background, and $E_{\mu}{ }^{\alpha}=$ $\delta_{\mu}{ }^{\alpha}$ at $\theta^{\mu}=0$, we may write the $\kappa$-symmetry transformation as

$$
\delta_{\kappa} \theta=\left[\Gamma^{a} \tilde{p}_{a}-(-1)^{p} \gamma\right] \kappa .
$$

This gauge transformation allows half the worldvolume fermions to be gauged away. The supersymmetry transformations of the remaining half take the form

$$
\delta_{\epsilon} \theta=\epsilon+\delta_{\kappa(\epsilon)} \theta
$$

where $\kappa(\epsilon)$ is now a specific matrix function of worldvolume fields acting on the constant spinor $\epsilon$. Its precise form depends on the $\kappa$-symmetry gauge choice and it has the effect that $\delta_{\epsilon} \theta=M \epsilon$ for some matrix $M$ [15]. The matrix $M$ depends on the worldvolume fields and hence, for static fields, on the worldspace coordinates. At any given point on worldspace it is a matrix for which half the eigenvalues vanish. This is due to the fact that, locally, the brane realizes half the supersymmetry non-linearly. The spinors $\epsilon$ corresponding to the linearly-realized supersymmetries are the eigenspinors of $M$ with zero eigenvalue. These are the supersymmetries preserved, locally, by the brane. The supersymmetries that are globally preserved correspond to those spinors $\epsilon$ that are eigenspinors of $M$ with vanishing eigenvalue for every point on worldspace. Thus a worldvolume configuration is supersymmetric if the equation $M(\sigma) \epsilon=0$ has solutions for constant $\epsilon$. This is equivalent to the equation $\delta_{\epsilon} \theta=0$.

We now note the identity 


$$
\left[\Gamma^{a} \tilde{p}_{a}+\gamma\right] \delta_{\kappa} \theta=\left[\left(\tilde{p}^{2}+\operatorname{det} m\right)-2\left(\partial_{i} x^{I} p_{I}\right) \gamma^{i} \gamma\right] \kappa
$$

where $\gamma^{i}=m^{i j} \partial_{j} x^{I} \Gamma_{I}$. The right hand side vanishes as a result of the Hamiltonian and worldspace diffeomorphism constraints. Using these constraints we deduce that $\delta_{\epsilon} \theta=0$ implies

$$
\left[\Gamma^{a} \tilde{p}_{a}+\gamma\right] \epsilon=0,
$$

which is an alternative form of the condition for preservation of supersymmetry that obviates the need to compute the matrix $M$. This is the Hamiltonian form of the supersymmetry condition derived in 16, 17] from the Lagrangian formulation. Observe that the matrix multiplying $\epsilon$ is precisely the matrix appearing in the $\{Q, Q\}$ anticommutator, as required for consistency because it is manifest from (21) that eigenspinors of this matrix with vanishing eigenvalue correspond to supersymmetries that are preserved by the p-brane configuration.

We now turn to the consequences of the algebra (21) for static configurations with $\tilde{p}_{I}=0$. Given the normalized spinor $\epsilon$ of the earlier discussion we deduce from the positivity of $\{Q, Q\}$ that

$$
-\tilde{p}_{0}-\nu \epsilon^{T} \Gamma_{\underline{0}} \gamma \epsilon \geq 0 .
$$

The first term on the left hand side is just $\nu \sqrt{\operatorname{det} m}$, by the Hamiltonian constraint. For the second term we have

$$
\nu \epsilon^{T} \Gamma_{\underline{0}} \gamma \epsilon=\left.\varphi\right|_{\xi}
$$

where $\left.\varphi\right|_{\xi}$ is the evaluation on a tangent p-plane $\xi$ of a p-form $\varphi$ that is formally identical to (2) but now with $\Gamma_{0}=\nu \Gamma_{0}$ and Dirac matrices $\Gamma_{I}$ satisfying $\left\{\Gamma_{I}, \Gamma_{J}\right\}=$ $2 g_{I J}$. Thus, 27) is equivalent to

$$
\left.\varphi\right|_{\xi} \leq \nu \operatorname{vol}_{\xi} .
$$

Setting $\tilde{p}_{I}=0$, it follows from (21) that

$$
2(Q \epsilon)^{2}=\int d^{p} \sigma\left[\mathcal{H}-\left.\left(i_{k} A+\varphi\right)\right|_{\xi}\right] .
$$

The terms in the integrand other than $\mathcal{H}$ constitute a central extension of the supertranslation algebra and it follows from general considerations that such extensions are topological. In other words, the p-form $i_{k} A+\varphi$ must be closed. Equivalently

$$
d \varphi=\mathcal{F},
$$

where $\mathcal{F}=-d i_{k} A$. A p-form $\varphi$ that satisfies both the inequality (29) and the condition (31) will be called a 'generalized calibration'. This definition is modeled on the definition of an 'adS' calibration in [9], and adS calibrations are examples of 'generalized' calibrations; we will later provide some further examples that are not adS calibrations.

It will now be shown that the calibrated manifolds of a generalization calibration are minimal worldspaces; the argument is a straightforward extension of the one given for adS calibrations in [9]. Let $U$ be an open subset of a calibrated manifold of a generalized calibration $\varphi$. Then

$$
\int_{U} d^{p} \sigma \nu \sqrt{\operatorname{det} m}=\int_{U} \varphi
$$

Now let $V$ be another open p-dimensional set in the same homology class as $U$ with $\partial V=\partial U$, and let $D$ be a $(\mathrm{p}+1)$-surface with $\partial D=U-V$. Then

$$
\int_{U} \varphi=\int_{V} \varphi+\int_{D} d \varphi
$$

Using (31) we have

$$
\int_{D} d \varphi=-\int_{D} d\left(i_{k} A\right)=\int_{V} d^{p} \sigma \Phi-\int_{U} d^{p} \sigma \Phi
$$

and hence

$$
\begin{aligned}
& \int_{U} d^{p} \sigma[\nu \sqrt{\operatorname{det} m}+\Phi]=\int_{V} \varphi+\int_{V} d^{p} \sigma \Phi \\
\leq & \int_{V} d^{p} \sigma[\nu \sqrt{\operatorname{det} m}+\Phi]
\end{aligned}
$$

where the inequality follows from (29). We conclude that

$$
E(U) \leq E(V)
$$

where $E$ is the energy, and hence that $U$, initially defined as a subset of the contact set of a generalized calibration, is also a subset of a minimal worldspace. Since $U$ is arbitrary we conclude that the entire calibrated manifold of a generalized calibration is a minimal worldspace, as claimed. We remark that if $d i_{k} A=0$, then the above bound of the generalized calibration reduces to the bound for calibrated manifolds saturated by minimal surfaces.

Before proceeding to the examples we should discuss one potential difficulty with the application of the above ideas. In many cases of interest, static branes are invariant not only under time translations but also under some number, $\ell$ say, of space translations. In fact, in these cases the invariance group is usually the Poincaré group of isometries of $\mathbb{E}^{(1, \ell)}$, in which case the brane configuration has an interpretation as an $\ell$-brane soliton on the p-dimensional worldspace, i.e. an $\ell$-brane 'worldvolume soliton'. For $\ell>0$, the energy density is independent of some directions and the total energy will be proportional to the volume of $\mathbb{E}^{\ell}$, which is infinite. This problem can be resolved by periodic identification of the $\ell$ coordinates. Alternatively, we may minimise the energy per unit $\ell$-volume, which we also denote by $E$. This is

$$
E=\int d^{p-\ell} \sigma\left[\nu_{\ell} \sqrt{\operatorname{det} m}+\Phi_{\ell}\right]
$$

where (for static spacetimes) $\nu_{\ell}=\sqrt{\operatorname{det} m_{\ell}}$, with $m_{\ell}$ being the induced metric on $\mathbb{E}^{(1, \ell)}$, and $m$ is now the induced metric on a section of the worldvolume with 
constant $\mathbb{E}^{(1, \ell)}$ coordinates. Similarly, $\Phi_{\ell}$ is induced by $i_{k_{0}} \ldots i_{k_{\ell}} A$ where $k_{0}, \ldots, k_{\ell}$ are the translational Killing vector fields of $\mathbb{E}^{(1, \ell)}$. This is the functional used in 9] to construct the adS calibration bound. For every configuration $B$ that minimizes the above energy functional, we find a p-brane solution with topology $\mathbb{E}^{(1, \ell)} \times B$. The considerations of supersymmetry that we have described above for the $\ell=0$ case can easily be extended to the $\ell>0$ cases. In particular, a generalized calibration satisfies

$$
\begin{aligned}
\left.\varphi\right|_{\xi} & \leq \nu_{\ell} \operatorname{vol}_{\xi} \\
d \varphi & =\mathcal{F}^{\ell}
\end{aligned}
$$

where $\mathcal{F}^{\ell}=-d i_{k_{0}} \ldots i_{k_{\ell}} A$.

\section{M2-BRANES AND GENERALIZED CALIBRATIONS}

We shall begin by discussing some examples of ordinary calibrations in non-flat backgrounds. This will allow us to provide an interpretation in terms of calibrations of some recent work on sigma-model lump solitons on the M2brane [8]; specifically we show that they correspond to calibrated 2-surfaces associated to Kähler calibrationsf. We will then turn to the cases associated with generalized calibrations.

The general setup for lumps on an M2-brane is a probe M2-brane in a supergravity background of the form $\mathbb{E}^{(1,2)} \times M_{8}$ with metric

$$
d s^{2}=d s^{2}\left(\mathbb{E}^{(1,2)}\right)+d s_{8}^{2} .
$$

We embed the worldvolume and fix the worldvolume reparameterizations such that the $\mathbb{E}^{(1,2)}$ coordinates are identified with the worldvolume coordinates. We then consider static worldvolume configurations $y^{a}=$ $\left.y^{a}\left(\sigma^{1}, \sigma^{2}\right)\right\}$, where $\left\{y^{a} ; a=1, \ldots 8\right\}$ are coordinates for $M_{8}$ and $\left(\sigma^{1}, \sigma^{2}\right)$ are the worldspace coordinates. The M2-brane energy is then

$$
E=\int d^{2} \sigma \sqrt{\operatorname{det} m}
$$

where $m$ is the metric induced from $d s_{8}^{2}$. We see that, in this case, the redshift factor $\nu$ is unity, and $\Phi$ vanishes.

The case considered in [8] was $M_{8}=\mathbb{E}^{4} \times M_{4}$ with a KK-monopole metric on $M_{4}$. This is a circle bundle over $\mathbb{E}^{3}$ with the circle degenerating to a point at the 'centres' of the metric, which are points in $\mathbb{E}^{3}$. The subcase considered in 8 was the two-centre metric, for which there is a privileged direction in $\mathbb{E}^{3}$ defined (up to a sign) by

\footnotetext{
${ }^{\dagger}$ Solutions to the Cayley calibration equations found in 18 were characterised there as 'octonionic lumps'.
}

the line between the two centres. Let $n$ be a unit vector in this direction. The metric is hyper-Kähler so there are three complex structures $I_{r}(r=1,2,3)$. In particular, the linear combination

$$
J=n^{r} I_{r}
$$

is a complex structure. The lump configuration of the M2-brane discussed in [8] is associated with the 2-form

$$
\varphi=d x^{1} \wedge d x^{2}+\omega_{J}
$$

where $\omega_{J}$ is the Kähler form of $J$ with respect to the hyper-Kähler metric on $M_{4}$. This is a Kähler form on $\mathbb{E}^{2} \times M_{4}$ and satisfies the inequality 29 . It is therefore a calibration. The calibration bound is

$$
E \geq|\varphi|
$$

which is saturated by the lump configurations. These are desingularized intersections of the M2-brane probe with an M2-brane wrapped on the finite area holomorphic 2cycle of the background. This cycle can be decribed as follows. Let $\theta$ be the $S^{1}$ coordinate for the $S^{1}$ fibre of $M_{4}$ and choose the centres in $\mathbb{E}^{3}$ of the $M_{4}$ metric to be at $u= \pm L$ on the $u$-axis. Then the 2-cycle is $\{\theta, u ;-L \leq$ $u \leq L\}$.

To find the energy of the lump soliton one must subtract the vacuum energy of the M2-brane probe. To see how this may be done we note that the energy of the lump is independent of its size, which is arbitrary. A zero size lump corresponds to a point intersection of the M2-brane probe with the wrapped M2-brane. The two M2-branes can then be moved apart, without changing the energy, so that they 'overlap' rather than intersect. We then have two separate M2-branes, each of which must be a calibrated surface. This is evident for the probe M2-brane, which is now an infinite planar membrane in $\mathbb{E}^{4}$; it is calibrated by the two-form $d x^{1} \wedge d x^{2}$ and its energy is the infinite vacuum energy. The energy of the lump soliton is therefore equal to that of the wrapped M2-brane. The energy is finite since the volume of this surface is finite. The surface itself, described above, is calibrated by the Kähler 2-form $\omega_{J}$.

Various generalizations of this construction are possible. A multi-centre metric has various finite area holomorphic 2-cycles around which an M2-brane may be wrapped. Intersections with another infinite planar M2brane are again, when desingularized, lump solitons, although there is now more than one type of lump. Another generalization arises from the fact that the 8-metric $d s_{8}^{2}$ could be any eight-dimensional Ricci-flat metric, although it must have reduced holonomy if the background is to preserve any supersymmetry. The possible reduced holonomy groups are $S U(2), S U(3), S U(4), G_{2}, \operatorname{Spin}(7)$ and $S p(2)$. For M2-brane probes in these backgrounds the only relevant calibrations are of degree two, and the supersymmetric backgrounds admitting a degree two calibration are those for which the holonomy group is $S U(2)$, 
$S U(3), S U(4)$ or $S p(2)$. All these cases admit Kähler calibrations; the calibrated surfaces are holomorphic twodimensional subspaces.

So far we have not needed the full power of generalized calibrations. These arise by placing M2-branes in a $\mathrm{D}=11$ supergravity background with non-vanishing 4-form field strength. An example is provided by an M2-brane probe placed parallel to a M2-brane background. The relevant calibrations that describe the supersymmetric dynamics of the probe are hermitian of degree two. The metric $m$ and the electrostatic potential $\Phi$ of (1) are induced via the map $x^{i}(\sigma)=\sigma^{i}, y^{a}=y^{a}(\sigma)$, from the metric $d s^{2} \equiv$ $g_{I J} d Y^{I} d Y^{J}$ and the 3 -form $\mathcal{F} \equiv-d i_{k} A$, respectively, where

$$
\begin{aligned}
d s^{2} & =H^{-\frac{2}{3}}\left[\left(d x^{1}\right)^{2}+\left(d x^{2}\right)^{2}\right]+H^{\frac{1}{3}} \delta_{a b} d y^{a} d y^{b} \\
\mathcal{F} & =d H^{-1} \wedge d x^{1} \wedge d x^{2}
\end{aligned}
$$

and $H$ is the familiar harmonic function of the multi M2brane supergravity solution. The redshift factor is $\nu=$ $H^{-\frac{1}{3}}$.

To define the Hermitian generalized calibration relevant to this case it suffices to give the Hermitian form of the complex structure on the 10 -space. This is

$$
\omega_{J}=H^{-1} d x^{1} \wedge d x^{2}+\omega_{K}
$$

where $\omega_{K}$ is a (constant) Kähler 2-form for $\mathbb{E}^{8}$. Note that $\mathcal{F}=d \omega_{J}$, as required for a generalized calibration. In addition, $\omega_{J}$ is the two-form obtained from the almost complex structure by raising an index with the rescaled metric

$$
d \tilde{s}^{2}=\nu d s^{2}=H^{-1}\left[\left(d x^{1}\right)^{2}+\left(d x^{2}\right)^{2}\right]+\delta_{a b} d y^{a} d y^{b} .
$$

The almost complex structure is constant and hence integrable. It then follows from Wirtinger's inequality that $\omega_{J}$ is a generalized calibration. The calibrated surfaces are holomophic curves in the M2-brane background. Note that the metric $\tilde{m}$ induced on worldspace by the rescaled metric $d \tilde{s}^{2}$ is, in this case, such that $\sqrt{\operatorname{det} \tilde{m}}=\nu \sqrt{\operatorname{det} m}$.

There are many holomorphic curves in the M2-brane background. For embeddings of the form $x^{i}=\sigma^{i}$, $y^{a}=y^{a}(\sigma)$, as above, they are roughly characterized by the number of 'active' transverse scalars. From the bulk perspective, every pair of active scalars is interpreted as the worldvolume coordinates of another M2-brane, so the bulk interpretation of the solution with $2 k$ active scalars is that of $k+1$ M2-branes intersecting, or overlapping, (not necessarily orthogonally) on a 0-brane soliton, all in the given M2-brane background. This interpretation suggests that the proportion of the 32 supersymmetries preserved by the probe M2-brane is $2^{-(k+1)}$, in agreement with the proportion derived using the contact set $S U(k+1) / S(U(1) \times U(k))$ of the calibration (note that for these examples the presence of the background does not affect the count of supersymmetries).

\section{M5-BRANES AND GENERALIZED CALIBRATIONS}

Lumps are also relevant to static M5-brane configurations. For this we place a M5-brane in a background of the form $\mathbb{E}^{(1,5)} \times M_{5}$ where the $M_{5}$ metric is Ricci flat and, for preservation of supersymmetry, has holonomy in $S U(2)$. It follows that $M_{5}=\mathbb{E} \times M_{4}$, with a direct product metric such that the $M_{4}$ metric has holonomy in $S U(2)$. The $S U(2)$ Kähler calibration of degree two discussed above is again applicable; the interpretation is as M5-branes intersecting on a 3-brane in a KKmonopole background. However, there are now other, higher-degree, calibrations to consider. One such case is a degree four Kähler calibration, which we now describe. Let $\left(x^{0}, x^{i}\right)(i=1,2,3,4,5)$ be cartesian cordinates for $\mathbb{E}^{(1,5)}$, and let $y^{a}(a=1, \ldots, 4)$ be coordinates on $M_{4}$. Consider the manifold $\mathbb{E}^{4} \times M_{4}$ where $\mathbb{E}^{4}$ is the subspace of $\mathbb{E}^{(1,5)}$ with constant $x^{0}$ and $x^{5}$. This is a Kähler manifold with Kähler 2-form

$$
\omega=d x^{1} \wedge d x^{2}+d x^{3} \wedge d x^{4}+\omega_{J},
$$

where $\omega_{J}$ is defined as for the case of the M2-brane. We fix the worldvolume diffeomorphisms, and partially specify the embedding of the M5-brane in spacetime, by choosing $x^{0}=t$ and identifying $x^{i}$ with the worldspace coordinates $\sigma^{i}$. In addition, we choose the four functions $y^{a}$ to be independent of both the time and one of the worldspace coordinates. This means that the M5-brane has topology $\mathbb{E}^{(1,1)} \times B$ where $B$ is a 4 -surface parameterised by the other four worldspace coordinates. The four functions $y^{a}$ define a minimal embedding of $B$ in $M_{4}$ associated to the calibration 4-form

$$
\varphi=\frac{1}{2} \omega \wedge \omega
$$

If we take $M_{4}=K_{3}$ then the M5-brane is wrapped on $K_{3}$ and can be identified with the heterotic string [19]. We have now seen that this wrapping can be described by saying that $B$ is a minimal 4 -surface in $K_{3}$ calibrated by an $S U(2)$ Kähler calibration of degree four.

Examples of generalized calibrations can be found by considering an M5-brane probe in an M5-brane supergravity background, for which the metric and seven-form field strength are respectively

$$
\begin{aligned}
d s^{2} & =H^{-\frac{1}{3}} d s^{2}\left(\mathbb{E}^{(1,5)}\right)+H^{\frac{2}{3}} d s^{2}\left(\mathbb{E}^{5}\right) \\
F & =-d H^{-1} \wedge d x^{0} \wedge d x^{1} \wedge \ldots \wedge d x^{5},
\end{aligned}
$$

where $H$ is a harmonic function on $\mathbb{E}^{5}$, which we choose to be

$$
H=1+\frac{\mu}{r^{3}}
$$

for positive constant $\mu$. We assume here that the selfdual 3-form field strength on the M5-brane vanishes; otherwise the theory of generalized calibrations elaborated 
above would be inapplicable. The Bianchi identity for this field strength then implies that the pull-back to the worldvolume of the four form $F$ must also vanish. This condition is not satisfied for five active scalars, so we consider only those cases with four or fewer active scalars 7 . There are still many more such cases than there were for the M2-brane. They include 'Hermitian', 'Special Almost Symplectic' (SAS) and 'exceptional' calibrations, generalizing the corresponding calibrations that have been investigated in the 'near-horizon' adS backgrounds [9]. The calibration bounds depends on the degree of the calibration and the number of active scalars. We are therefore led to an organisation in which we first specify the number of active scalars and then consider the various degrees for the calibration form. In what follows $\left\{x^{1}, \ldots, x^{5-\ell}\right\}$ will denote the worldvolume coordinates of the M5-brane which are transverse to the $\ell$-brane worldvolume soliton and $\left\{y^{1}, \ldots, y^{n}\right\}, n \leq 5$, will denote the transverse scalars. As in the M2-brane case, it is convenient to consider a rescaled metric. Taking this rescaling into account, we have

$$
\begin{aligned}
d \tilde{s}^{2} & =\nu_{\ell}^{\frac{1}{5-\ell}}\left[H^{-\frac{1}{3}} d s^{2}\left(\mathbb{E}^{(5-\ell)}\right)+H^{\frac{2}{3}} d s^{2}\left(\mathbb{E}^{n}\right)\right] \\
\mathcal{F}^{\ell} & =d H^{-1} \wedge d x^{1} \wedge \ldots \wedge d x^{5-\ell}
\end{aligned}
$$

where $\nu_{\ell}=H^{-\frac{\ell+1}{3}}$. The static solutions will be M5branes with topology $\mathbb{E}^{(1, \ell)} \times B$, where $B$ is a calibrated surface of dimension $p-\ell$. We now consider the various subcases in turn.

\section{A. Hermitian M5-brane Calibrations}

There are four hermitian calibrations relevant to the M5-brane which are best distinguished by the group that rotates the complex planes in their contact set. In all cases, the calibrated submanifold $B$ is a holomorphic submanifold of the M5-brane background.

$S U(2)$ hermitian calibrations: These are degree two calibrations with two transverse scalars. The relevant four-dimensional metric and form field strength are

$$
\begin{aligned}
d \widetilde{s}^{2} & =H^{-1}\left[\left(d x^{1}\right)^{2}+\left(d x^{2}\right)^{2}\right]+\left(d y^{1}\right)^{2}+\left(d y^{2}\right)^{2} \\
\mathcal{F}^{3} & =d H^{-1} \wedge d x^{1} \wedge d x^{2}
\end{aligned}
$$

respectively. The calibration 2 -form is

$$
\varphi=H^{-1} d x^{1} \wedge d x^{2}+d y^{1} \wedge d y^{2},
$$

which is the Hermitian form of a complex structure. The complex coordinates are $\left\{z^{1}=y^{1}+i y^{2}, z^{2}=x^{1}+i x^{2}\right\}$

\footnotetext{
${ }^{\ddagger}$ For a multi-centre M5-brane solution we would need further restrictions on the number of active transverse scalars in order to satisfy this condition.
}

and the calibrated submanifold is given by the vanishing locus of a holomorphic function $f\left(z^{1}, z^{2}\right)$. The 'Killing' spinors obey the conditions

$$
\begin{aligned}
\Gamma_{0} \Gamma_{1} \Gamma_{2} \Gamma_{3} \Gamma_{4} \Gamma_{5} \epsilon & =\epsilon \\
\Gamma_{1} \Gamma_{2} \epsilon & =\Gamma_{6} \Gamma_{7} \epsilon .
\end{aligned}
$$

where the gamma matrices are in an orthonormal basis. We conclude that the solution preserves $\frac{1}{4}$ of bulk supersymmetry ${ }^{3}$. The bulk interpretation of the solutions is that of two M5-branes intersecting (at $S U(2)$ angles) on a 3-brane, with one of them parallel to the background.

$S U(3)$ hermitian calibrations: There are two cases to consider. The first is a degree two calibration with four transverse scalars. The relevant metric and form field strength are, respectively,

$$
\begin{aligned}
d \tilde{s}^{2} & =H^{-1}\left[\left(d x^{1}\right)^{2}+\left(d x^{2}\right)^{2}\right] \\
& +\left(d y^{1}\right)^{2}+\left(d y^{2}\right)^{2}+\left(d y^{3}\right)^{2}+\left(d y^{4}\right)^{2} \\
\mathcal{F}^{3} & =d H^{-1} \wedge d x^{1} \wedge d x^{2} .
\end{aligned}
$$

The calibration two-form is

$$
\varphi=H^{-1} d x^{1} \wedge d x^{2}+d y^{1} \wedge d y^{2}+d y^{3} \wedge d y^{4}
$$

which is again the Hermitian form of a complex structure. The holomorphic coordinates are $\left\{z^{1}=y^{1}+i y^{2}, z^{2}=\right.$ $\left.y^{3}+i y^{4}, z^{3}=x^{1}+i x^{2}\right\}$ and the calibrated surfaces can be described as the vanishing locus of two holomorphic functions $f^{1}\left(z^{1}, z^{2}, z^{3}\right), f^{2}\left(z^{1}, z^{2}, z^{3}\right)$. The Killing spinors satisfy the conditions

$$
\begin{aligned}
\Gamma_{0} \Gamma_{1} \Gamma_{2} \Gamma_{3} \Gamma_{4} \Gamma_{5} \epsilon & =\epsilon \\
\Gamma_{1} \Gamma_{2} \epsilon & =\Gamma_{6} \Gamma_{7} \epsilon \\
\Gamma_{1} \Gamma_{2} \epsilon & =\Gamma_{8} \Gamma_{9} \epsilon,
\end{aligned}
$$

from which we deduce that the solution preserves $\frac{1}{8}$ of (bulk) supersymmetry. The bulk interpretation of the configuration is as intersecting M5-branes at $S U(3)$ angles for which the corresponding orthogonal intersection is

$$
\begin{array}{ll}
M 5 & 0,1,2,3,4,5, *, *, *, * \\
M 5 & 0, *, *, 3,4,5,6,7, *, * \\
M 5 & 0, *, *, 3,4,5, *, *, 8,9 .
\end{array}
$$

The other $\mathrm{SU}(3)$ hermitian calibration is a degree four calibration with two transverse scalars. The relevant metric and form field strength are

\footnotetext{
$\S^{\S}$ The first of these conditions can be interpreted as due either to the background or to the probe, so the examples being considered are those for which the background does not impose additional constraints.
} 


$$
\begin{aligned}
d \tilde{s}^{2} & =H^{-\frac{1}{2}}\left[\left(d x^{1}\right)^{2}+\left(d x^{2}\right)^{2}+\left(d x^{3}\right)^{2}+\left(d x^{4}\right)^{2}\right] \\
& +H^{\frac{1}{2}}\left[\left(d y^{1}\right)^{2}+\left(d y^{2}\right)^{2}\right] \\
\mathcal{F}^{1} & =d H^{-1} \wedge d x^{1} \wedge d x^{2} \wedge d x^{3} \wedge d x^{4}
\end{aligned}
$$

respectively. To describe the calibration form, we introduce the Hermitian form

$$
\omega=H^{-\frac{1}{2}}\left[d x^{1} \wedge d x^{2}+d x^{3} \wedge d x^{4}\right]+H^{\frac{1}{2}} d y^{1} \wedge d y^{2} .
$$

The corresponding complex structure can be found by raising an index with the metric defined by $d \tilde{s}^{2}$. The calibration form is $\varphi=\frac{1}{2} \omega \wedge \omega$. In particular,

$$
\begin{aligned}
\varphi & =H^{-1} d x^{1} \wedge d x^{2} \wedge d x^{3} \wedge d x^{4} \\
& +d x^{1} \wedge d x^{2} \wedge d y^{1} \wedge d y^{2} \\
& +d x^{3} \wedge d x^{4} \wedge d y^{1} \wedge d y^{2}
\end{aligned}
$$

The holomorphic coordinates are $\left\{z^{1}=y^{1}+i y^{2}, z^{2}=\right.$ $\left.y^{3}+i y^{4}, z^{3}=x^{1}+i x^{2}\right\}$ and the calibrated surfaces can be described as the vanishing locus of a holomorphic function $f\left(z^{1}, z^{2}, z^{3}\right)$. The Killing spinors satisfy the conditions

$$
\begin{aligned}
\Gamma_{0} \Gamma_{1} \Gamma_{2} \Gamma_{3} \Gamma_{4} \Gamma_{5} \epsilon & =\epsilon \\
\Gamma_{1} \Gamma_{2} \epsilon & =\Gamma_{6} \Gamma_{7} \epsilon \\
\Gamma_{3} \Gamma_{4} \epsilon & =\Gamma_{6} \Gamma_{7} \epsilon,
\end{aligned}
$$

so the solution preserves $\frac{1}{8}$ of the bulk supersymmetry. The bulk interpretation of the configuration is as intersecting M5-branes at $S U(3)$ angles for which the corresponding orthogonal intersection is

$$
\begin{array}{ll}
M 5 & 0,1,2,3,4,5, *, * \\
M 5 & 0, *, *, 3,4,5,6,7 \\
M 5 & 0,1,2, *, *, 5,6,7 .
\end{array}
$$

$S U(4)$ hermitian calibration: The calibration of interest from the M5-brane perspective is the degree four calibration with four transverse scalars. The relevant metric and form field strength are, respectively,

$$
\begin{aligned}
d \tilde{s}^{2} & =H^{-\frac{1}{2}}\left[\left(d x^{1}\right)^{2}+\left(d x^{2}\right)^{2}+\left(d x^{3}\right)^{2}+\left(d x^{4}\right)^{2}\right] \\
& +H^{\frac{1}{2}}\left[\left(d y^{1}\right)^{2}+\left(d y^{2}\right)^{2}+\left(d y^{3}\right)^{2}+\left(d y^{4}\right)^{2}\right] . \\
\mathcal{F}^{1} & =d H^{-1} \wedge d x^{1} \wedge d x^{2} \wedge d x^{3} \wedge d x^{4}
\end{aligned}
$$

To describe the calibration form we introduce the Hermitian form

$$
\begin{aligned}
\omega & =H^{-\frac{1}{2}}\left[d x^{1} \wedge d x^{2}+d x^{3} \wedge d x^{4}\right] \\
& +H^{\frac{1}{2}}\left[d y^{1} \wedge d y^{2}+d y^{3} \wedge d y^{4}\right] .
\end{aligned}
$$

Then the calibration four-form is $\varphi=\frac{1}{2} \omega \wedge \omega$, i.e.

$$
\begin{aligned}
\varphi & =H^{-1} d x^{1} \wedge d x^{2} \wedge d x^{3} \wedge d x^{4} \\
& +\left[d x^{1} \wedge d x^{2} \wedge d y^{1} \wedge d y^{2}\right. \\
& +d x^{1} \wedge d x^{2} \wedge d y^{3} \wedge d y^{4} \\
& +d x^{3} \wedge d x^{4} \wedge d y^{1} \wedge d y^{2} \\
& \left.+d x^{3} \wedge d x^{4} \wedge d y^{3} \wedge d y^{4}\right] \\
& +H d y^{1} \wedge d y^{2} \wedge d y^{3} \wedge d y^{4}
\end{aligned}
$$

The complex coordinates are $\left\{z^{1}=y^{1}+i y^{2}, z^{2}=y^{3}+\right.$ $\left.i y^{4}, z^{3}=x^{1}+i x^{2}, z^{4}=x^{3}+i x^{4}\right\}$ and the calibrated manifolds can be described as the vanishing locus of two holomorphic functions $f^{1}\left(z^{1}, z^{2}, z^{3}, z^{4}\right), f^{2}\left(z^{1}, z^{2}, z^{3}, z^{4}\right)$. The conditions on the Killing spinors are

$$
\begin{aligned}
\Gamma_{0} \Gamma_{1} \Gamma_{2} \Gamma_{3} \Gamma_{4} \Gamma_{5} \epsilon & =\epsilon \\
\Gamma_{1} \Gamma_{2} \epsilon & =\Gamma_{6} \Gamma_{7} \epsilon \\
\Gamma_{3} \Gamma_{4} \epsilon & =\Gamma_{6} \Gamma_{7} \epsilon \\
\Gamma_{6} \Gamma_{7} \epsilon & =\Gamma_{8} \Gamma_{9} \epsilon,
\end{aligned}
$$

from which we deduce that the solution preserves $\frac{1}{16}$ of the bulk supersymmetry.

In all of these cases it is clear that $\mathcal{F}^{\ell}=d \varphi$, as required for consistency. In addition, in all the above cases an alternative interpretation of the solutions is as M5-branes with topology $\mathbb{E}^{(1, \ell)} \times C$, for either $\ell=1$ or $\ell=3$, where $C$ is a $(5-\ell)$-dimensional holomorphic cycle.

\section{B. SAS Calibrations}

The Special Almost Symplectic calibrations (SAS) of $A d S_{7} \times S^{4}$, which is the near horizon geometry of the M5-brane, have a natural generalization in the full M5brane background. The SAS calibrations associated with a M5-brane probe placed parallel to a M5-brane supergravity background have degrees two, three, or four (a calibration of degree five would have to involve the 2form tensor field and is therefore excluded). The degree two SAS calibration is identical to the degree two Hermitian calibration explained in the previous section. It therefore remains to describe the SAS calibrations with degrees three and four. For SAS calibrations the number of transverse scalars is the same as the degree of the calibration. So the degree three SAS calibration is a three-dimensional submanifold in a six-dimensional manifold and the degree four calibration is a four-dimensional submanifold in a eight-dimensional manifold.

For the degree three SAS calibration, the relevant metric and form field strength are

$$
\begin{aligned}
d \tilde{s}^{2} & =H^{-\frac{2}{3}}\left[\left(d x^{1}\right)^{2}+\left(d x^{2}\right)^{2}+\left(d x^{3}\right)^{2}\right] \\
& +H^{\frac{1}{3}}\left[\left(d y^{1}\right)^{2}+\left(d y^{2}\right)^{2}+\left(d y^{3}\right)^{2}\right] \\
\mathcal{F}^{2} & =d H^{-1} \wedge d x^{1} \wedge d x^{2} \wedge d x^{3}
\end{aligned}
$$

respectively.

To proceed we introduce the orthonormal frame

$$
\begin{aligned}
e^{p} & =H^{-\frac{1}{3}} d x^{p}, & & 1 \leq p \leq 3 \\
e^{3+p} & =H^{\frac{1}{6}} d y^{p}, & & 1 \leq p \leq 3
\end{aligned}
$$

The almost symplectic form is defined as

$$
\omega \equiv \sum_{p=1}^{3} e^{p} \wedge e^{3+p}=H^{-\frac{1}{6}} \sum_{i=1}^{3} d x^{i} \wedge d y^{i} .
$$


Using the almost complex structure associated with this almost symplectic form, the calibration form is found to be

$$
\begin{aligned}
\varphi & \equiv \operatorname{Re}\left(e^{1}+i e^{4}\right) \wedge \ldots \wedge\left(e^{3}+i e^{6}\right) \\
& =H^{-1} d x^{1} \wedge d x^{2} \wedge d x^{3}-d x^{1} \wedge d y^{2} \wedge d y^{3} \\
& -d y^{1} \wedge d x^{2} \wedge d y^{3}-d y^{1} \wedge d y^{2} \wedge d x^{3}
\end{aligned}
$$

Repeating the same arguments as in the AdS case in [9], the calibrated submanifolds are seen to be determined by a single real function $f\left(x^{1}, x^{2}, x^{3}\right)$ such that

$$
\begin{aligned}
y^{i} & =\frac{\partial}{\partial x^{i}} f\left(x^{1}, x^{2}, x^{3}\right) \\
H^{-1} \delta^{m n} \partial_{m} \partial_{n} f & =\operatorname{det}\left(\partial_{i} \partial_{j} f\right) .
\end{aligned}
$$

The Killing spinors of such solutions satisfy the conditions

$$
\begin{aligned}
& \Gamma_{0} \Gamma_{1} \Gamma_{2} \Gamma_{3} \Gamma_{4} \Gamma_{5} \epsilon=\epsilon \\
& \Gamma_{0} \Gamma_{3} \Gamma_{4} \Gamma_{5} \Gamma_{6} \Gamma_{7} \epsilon=-\epsilon \\
& \Gamma_{0} \Gamma_{2} \Gamma_{4} \Gamma_{5} \Gamma_{6} \Gamma_{8} \epsilon=\epsilon,
\end{aligned}
$$

and so the configuration preserves $\frac{1}{8}$ of the bulk supersymmetry. The bulk interpretation of the solution is that of three intersecting M5-branes, possibly at $S U(3)$ angles, for which the associated orthogonal intersection is

$$
\begin{array}{ll}
M 5 & 0,1,2,3,4,5, *, *, * \\
\overline{M 5} & 0, *, *, 3,4,5,6,7, * \\
M 5 & 0, *, 2, *, 4,5,6, *, 8
\end{array}
$$

Alternatively, the solution describes a M5-brane with topology $\mathbb{E}^{(1,2)} \times B$, where $B$ is a three-dimensional SAS calibrated submanifold.

For the degree four calibration, the relevant metric and form field strength are

$$
\begin{aligned}
d \tilde{s}^{2} & =H^{-\frac{1}{2}}\left[\left(d x^{1}\right)^{2}+\left(d x^{2}\right)^{2}+\left(d x^{3}\right)^{2}+\left(d x^{4}\right)^{2}\right] \\
& +H^{\frac{1}{2}}\left[\left(d y^{1}\right)^{2}+\left(d y^{2}\right)^{2}+\left(d y^{3}\right)^{2}+\left(d y^{4}\right)^{2}\right] \\
\mathcal{F}^{1} & =d H^{-1} \wedge d x^{1} \wedge \ldots \wedge d x^{4}
\end{aligned}
$$

respectively. We again introduce the frame

$$
\begin{aligned}
e^{p} & =H^{-\frac{1}{4}} d x^{p}, & & 1 \leq p \leq 4 \\
e^{4+p} & =H^{\frac{1}{4}} d y^{p}, & & 1 \leq p \leq 4,
\end{aligned}
$$

and the symplectic form

$$
\omega \equiv \sum_{p=1}^{4} e^{p} \wedge e^{4+p}=\sum_{i=1}^{3} d x^{i} \wedge d y^{i} .
$$

Using again the almost complex structure associated with $\omega$, the calibration form is found to be

$$
\begin{aligned}
\varphi & =\operatorname{Re}\left(e^{1}+i e^{4}\right) \wedge \ldots \wedge\left(e^{4}+i e^{8}\right) \\
& =H^{-1} d x^{1} \wedge d x^{2} \wedge d x^{3} \wedge d x^{4} \\
& -d x^{1} \wedge d x^{2} \wedge d y^{3} \wedge d y^{4} \\
& -d x^{1} \wedge d y^{2} \wedge d x^{3} \wedge d y^{4} \\
& -d x^{1} \wedge d y^{2} \wedge d y^{3} \wedge d x^{4} \\
& -d y^{1} \wedge d y^{2} \wedge d x^{3} \wedge d x^{4} \\
& -d y^{1} \wedge d x^{2} \wedge d y^{3} \wedge d x^{4} \\
& -d y^{1} \wedge d x^{2} \wedge d x^{3} \wedge d y^{4} \\
& +H d y^{1} \wedge d y^{2} \wedge d y^{3} \wedge d y^{4} .
\end{aligned}
$$

As in the previous case, the solutions are determined by a single real function $f\left(x^{1}, x^{2}, x^{3}, x^{4}\right)$ such that

$$
\begin{aligned}
y^{i} & =\frac{\partial}{\partial x^{i}} f\left(x^{1}, x^{2}, x^{3}, x^{4}\right) \\
H^{-1} \delta^{m n} \partial_{m} \partial_{n} f & =\sum_{m} \operatorname{det}_{m \mid m}\left(\partial_{i} \partial_{j} f\right)
\end{aligned}
$$

where $\operatorname{det}_{m \mid m}$ denotes the determinant of a matrix with the m-th row and column omitted. The Killing spinors of these solutions obey the conditions

$$
\begin{aligned}
\Gamma_{0} \Gamma_{1} \Gamma_{2} \Gamma_{3} \Gamma_{4} \Gamma_{5} \epsilon & =\epsilon \\
\Gamma_{1} \Gamma_{2} \epsilon & =-\Gamma_{6} \Gamma_{7} \epsilon \\
\Gamma_{1} \Gamma_{3} \epsilon & =-\Gamma_{6} \Gamma_{8} \epsilon \\
\Gamma_{1} \Gamma_{4} \epsilon & =-\Gamma_{6} \Gamma_{9} \epsilon
\end{aligned}
$$

and so they preserve $\frac{1}{16}$ of the bulk supersymmetry. The bulk interpretation of the solutions is as four intersecting M5-branes, possibly at $S U(4)$ angles, for which the corresponding orthogonal intersection is

$$
\begin{array}{ll}
M 5 & 0,1,2,3,4,5, *, *, *, * \\
\overline{M 5} & 0, *, *, 3,4,5,6,7, *, * \\
M 5 & 0, *, 2, *, 4,5,6, *, 8, * \\
\overline{M 5} & 0, *, 2,3, *, 5,6, *, *, 9 .
\end{array}
$$

Alternatively, the solution describes a M5-brane with topology $\mathbb{E}^{(1,1)} \times B$, where $B$ is a four-dimensional SAS submanifold.

\section{Exceptional Calibrations}

There are three exceptional calibrations to consider as follows: The Cayley calibration which is a degree four calibration with four transverse scalars. The relevant eight-dimensional metric and form field strength are

$$
\begin{aligned}
d \tilde{s}^{2} & =H^{-\frac{1}{2}}\left[\left(d x^{1}\right)^{2}+\left(d x^{2}\right)^{2}+\left(d x^{3}\right)^{2}+\left(d x^{4}\right)^{2}\right] \\
& +H^{\frac{1}{2}}\left[\left(d y^{1}\right)^{2}+\left(d y^{2}\right)^{2}+\left(d y^{3}\right)^{2}+\left(d y^{4}\right)^{2}\right] \\
\mathcal{F}^{1} & =d H^{-1} \wedge d x^{1} \wedge \ldots \wedge d x^{4}
\end{aligned}
$$

respectively. The calibration four-form can be constructed from the metric and a $\operatorname{Spin}(7)$ invariant self-dual four-form on $\mathbb{E}^{8}$, viz 


$$
\begin{aligned}
\varphi & =H^{-1} d x^{1} \wedge d x^{2} \wedge d x^{3} \wedge d x^{4}+\left[d x^{3} \wedge d x^{4} \wedge d y^{3} \wedge d y^{4}\right. \\
& +d x^{2} \wedge d x^{4} \wedge d y^{2} \wedge d y^{4}+d x^{2} \wedge d x^{3} \wedge d y^{2} \wedge d y^{3} \\
& +d x^{1} \wedge d x^{3} \wedge d y^{2} \wedge d y^{4}+d x^{1} \wedge d x^{2} \wedge d y^{1} \wedge d y^{2} \\
& +d x^{1} \wedge d x^{3} \wedge d y^{1} \wedge d y^{3}+d x^{1} \wedge d x^{4} \wedge d y^{1} \wedge d y^{4} \\
& +d x^{2} \wedge d x^{4} \wedge d y^{1} \wedge d y^{3}-d x^{1} \wedge d x^{2} \wedge d y^{3} \wedge d y^{4} \\
& -d x^{1} \wedge d x^{4} \wedge d y^{2} \wedge d y^{3}-d x^{3} \wedge d x^{4} \wedge d y^{1} \wedge d y^{2} \\
- & \left.d x^{2} \wedge d x^{3} \wedge d y^{1} \wedge d y^{4}\right] \\
& +H d y^{1} \wedge d y^{2} \wedge d y^{3} \wedge d y^{4}
\end{aligned}
$$

The equations which the transverse scalars satisfy are

$$
\begin{aligned}
H^{-1}\left(\partial_{1} Y\right. & \left.-\partial_{2} Y i-\partial_{3} Y j-\partial_{4} Y k\right)=\partial_{2} Y \times \partial_{3} Y \times \partial_{4} Y \\
& +\partial_{1} Y \times \partial_{3} Y \times \partial_{4} Y i-\partial_{1} Y \times \partial_{2} Y \times \partial_{4} Y j \\
& +\partial_{1} Y \times \partial_{2} Y \times \partial_{3} Y k
\end{aligned}
$$

together with the second order auxiliary condition

$$
\begin{aligned}
\operatorname{Im} & {\left[\left(\partial_{1} Y \times \partial_{2} Y-\partial_{3} Y \times \partial_{4} Y\right) i\right.} \\
& +\left(\partial_{1} Y \times \partial_{3} Y+\partial_{2} Y \times \partial_{4} Y\right) j \\
& \left.+\left(\partial_{1} Y \times \partial_{4} Y-\partial_{2} Y \times \partial_{3} Y\right) k\right]=0,
\end{aligned}
$$

where $Y=y^{1}+i y^{2}+j y^{3}+k y^{4}, a \times b \times c=\frac{1}{2}(a \bar{b} c-c \bar{b} a)$, $a \times b=-\frac{1}{2}(\bar{a} b-\bar{b} a)$ and $i, j, k$ are the imaginary unit quaternions. The Killing spinors satisfy the conditions

$$
\begin{aligned}
\Gamma_{0} \Gamma_{1} \Gamma_{2} \Gamma_{3} \Gamma_{4} \Gamma_{5} \epsilon & =\epsilon \\
\Gamma_{3} \Gamma_{4} \epsilon & =-\Gamma_{8} \Gamma_{9} \epsilon \\
\Gamma_{2} \Gamma_{3} \epsilon & =-\Gamma_{7} \Gamma_{8} \epsilon \\
\Gamma_{1} \Gamma_{2} \epsilon & =-\Gamma_{6} \Gamma_{7} \epsilon \\
\Gamma_{1} \Gamma_{4} \epsilon & =\Gamma_{7} \Gamma_{8} \epsilon,
\end{aligned}
$$

so the solution preserves $\frac{1}{32}$ of bulk supersymmetry.

The Associative Calibration is a degree three calibration with four transverse scalars. The relevant sevendimensional metric and form field strength are

$$
\begin{aligned}
d \tilde{s}^{2} & =H^{-\frac{2}{3}}\left(\left(d x^{1}\right)^{2}+\left(d x^{2}\right)^{2}+\left(d x^{3}\right)^{2}\right) \\
& +H^{\frac{1}{3}}\left(\left(d y^{1}\right)^{2}+\left(d y^{2}\right)^{2}+\left(d y^{3}\right)^{2}+\left(d y^{4}\right)^{2}\right) \\
\mathcal{F}^{2} & =d H^{-1} \wedge d x^{1} \wedge d x^{2} \wedge d x^{3},
\end{aligned}
$$

respectively The calibration three-form is constructed from the above metric and the structure constants of octonions as follows:

$$
\begin{aligned}
\varphi & =H^{-1} d x^{1} \wedge d x^{2} \wedge d x^{3}+\left[d x^{1} \wedge d y^{1} \wedge d y^{2}\right. \\
& +d x^{3} \wedge d y^{1} \wedge d y^{4}+d x^{2} \wedge d y^{2} \wedge d y^{4} \\
& +d x^{2} \wedge d y^{1} \wedge d y^{3}-d x^{1} \wedge d y^{3} \wedge d y^{4} \\
& \left.-d x^{3} \wedge d y^{2} \wedge d y^{3}\right] .
\end{aligned}
$$

The equations which the transverse scalars satisfy are

$$
-H^{-1}\left(\partial_{1} Y i+\partial_{2} Y j+\partial_{3} Y k\right)=\partial_{1} Y \times \partial_{2} Y \times \partial_{3} Y,
$$

where $Y$ is defined as in the Cayley case. The conditions on the Killing spinor are

$$
\begin{aligned}
\Gamma_{0} \Gamma_{1} \Gamma_{2} \Gamma_{3} \Gamma_{4} \Gamma_{5} \epsilon & =\epsilon \\
\Gamma_{2} \Gamma_{3} \epsilon & =-\Gamma_{8} \Gamma_{9} \epsilon \\
\Gamma_{1} \Gamma_{3} \epsilon & =-\Gamma_{7} \Gamma_{9} \epsilon \\
\Gamma_{1} \Gamma_{3} \epsilon & =-\Gamma_{6} \Gamma_{8} \epsilon,
\end{aligned}
$$

so this configuration preserves $\frac{1}{16}$ of the bulk supersymmetry.

The Coassociative Calibration is a degree four calibration with three transverse scalars. The relevant sevendimensional metric and form field strength are

$$
\begin{aligned}
d \tilde{s}^{2} & =H^{-\frac{1}{2}}\left(\left(d x^{1}\right)^{2}+\left(d x^{2}\right)^{2}+\left(d x^{3}\right)^{2}+\left(d x^{4}\right)^{2}\right) \\
& +H^{\frac{1}{2}}\left(\left(d y^{1}\right)^{2}+\left(d y^{2}\right)^{2}+\left(d y^{3}\right)^{2}\right) \\
\mathcal{F}^{1} & =d H^{-1} \wedge d x^{1} \wedge \ldots \wedge d x^{4}
\end{aligned}
$$

respectively. The calibration four-form is constructed from the above metric and the dual form of the structure constants of octonions in $\mathbb{E}^{7}$ as

$$
\begin{aligned}
\varphi & =H^{-1} d x^{1} \wedge d x^{2} \wedge d x^{3} \wedge d x^{4} \\
& +\left[d x^{3} \wedge d x^{4} \wedge d y^{2} \wedge d y^{3}+d x^{2} \wedge d x^{4} \wedge d y^{1} \wedge d y^{3}\right. \\
& +d x^{2} \wedge d x^{3} \wedge d y^{1} \wedge d y^{2}+d x^{1} \wedge d x^{3} \wedge d y^{1} \wedge d y^{3} \\
& \left.-d x^{1} \wedge d x^{2} \wedge d y^{2} \wedge d y^{3}-d x^{1} \wedge d x^{4} \wedge d y^{1} \wedge d y^{2}\right]
\end{aligned}
$$

The transverse scalars of this calibration satisfy the equations

$$
-H^{-1}\left(\partial y^{1} i+\partial y^{2} j+\partial y^{3} k\right)=\partial y^{1} \times \partial y^{2} \times \partial y^{3},
$$

where $\partial=\partial_{1}+i \partial_{2}+j \partial_{3}+k \partial_{4} ; \partial_{i}=\partial / \partial x^{i}$. The conditions on the Killing spinors are

$$
\begin{aligned}
\Gamma_{0} \Gamma_{1} \Gamma_{2} \Gamma_{3} \Gamma_{4} \Gamma_{5} \epsilon & =\epsilon \\
\Gamma_{3} \Gamma_{4} \epsilon & =-\Gamma_{7} \Gamma_{8} \epsilon \\
\Gamma_{1} \Gamma_{2} \epsilon & =\Gamma_{7} \Gamma_{8} \epsilon \\
\Gamma_{2} \Gamma_{4} \epsilon & =-\Gamma_{6} \Gamma_{8} \epsilon,
\end{aligned}
$$

so the solutions preserve $\frac{1}{16}$ of the bulk supersymmetry. In all the above three types of calibration, $\mathcal{F}^{\ell}=d \varphi$ as required for consistency.

\section{CONCLUSIONS}

We have shown that generalized calibrations arise naturally in the context of supersymmetric configurations of p-brane actions. In particular, we have derived the generalized calibration bound from the p-brane supersymmetry algebra. We have also presented several examples of such calibrations in M-brane backgrounds. These examples by no means exhaust the possibilities. An obvious generalization would be to consider $1 / 4$ supersymmetric, 
intersecting-brane, backgrounds determined by two harmonic functions. More generally still, one can take any supersymmetric intersecting brane configuration in flat spacetime and replace one of the participating branes by its corresponding supergravity solution. One then expects to recover the full intersecting brane configuration as a generalized calibration of a probe brane in this background. Our examples are exclusively M-theoretic but string theory examples can easily be found and many are simply related to those discussed here by some duality chain.

The main limitation of calibrations is that the theory is inapplicable in those cases for which there are 'active' worldvolume vectors or tensors, as can happen for string theory D-branes and the M5-brane. Here again, however, many such cases are related to calibrations via duality chains so this limitation is not as severe as might be thought. We should point out that calibrations were discussed in the D-brane context in 20]. There is also the posibility that a reformulation of $\mathrm{D}$-branes and the M5-brane in which worldvolume vectors and tensors are induced from an extended spacetime, as recently proposed [21], might allow a direct extension of the theory of calibrations to these cases too.

Acknowledgments: J.G. thanks EPSRC for a studentship. G.P. is supported by a University Research Fellowship from the Royal Society. We are grateful to Jerome Gauntlett for helpful discussions.

[1] J.H. Hughes, J. Liu and J. Polchinski, Supermembranes, Phys. Lett. 180B (1986) 370.

[2] E. Bergshoeff, E. Sezgin and P.K. Townsend, Supermembranes and 11 dimensional supergravity, Phys. Lett. 189B (1987) 75.

[3] A. Achúcarro, J. Evans, P.K. Townsend and D. Wiltshire, Super p-branes, Phys. Lett. 198B (1987) 441.

[4] R. Harvey and H.B. Lawson, Calibrated geometries, Acta. Math. 148 (1982) 47.

[5] J. Dadok and R. Harvey, Calibrations and Spinors, Acta Math. 170 (1993) 83.

[6] G.W. Gibbons and G. Papadopoulos, Calibrations and intersecting branes, Commun. Math. Phys. 202 (1999) 593 ;

J.P. Gauntlett, N.D. Lambert and P.C. West, Branes and calibrated geometries, Commun. Math. Phys. 202 (1999) 571 ;

B.S. Acharya, J.M. Figueroa-O'Farrill and B. Spence, Branes at angles and calibrations, JHEP 04:012 (1998).

[7] K. Becker, M. Becker, D.R. Morrison, H. Ooguri, Y. Oz and Z. Yin, Supersymmetric cycles in exceptional holon- omy manifolds and Calabi-Yau 4-folds, Nucl. Phys. B480 (1996) 225.

[8] E. Bergshoeff and P.K. Townsend, Solitons on the Supermembrane, JHEP 05:021 (1999).

[9] J. Gutowski and G. Papadopoulos, adS Calibrations, hep-th/9902034.

[10] J. A. de Azcárraga, J.P. Gauntlett, J.M. Izquierdo and P.K. Townsend, Topological extensions of the supersymmetry algebra for extended objects, Phys. Rev. Lett. 63 (1989) 2443.

[11] T. Sato, Superalgebras in many types of M-brane backgrounds and various supersymmetric brane configurations, Nucl. Phys. B548 (1999) 231.

[12] E. Bergshoeff, E. Sezgin and Y. Tanii, Hamiltonian formulation of the supermembrane, Nucl. Phys. B298 (1988) 187.

[13] J.A. de Azcárraga, J.M. Izquierdo and P.K. Townsend, Classical anomalies of supersymmetric extended objects, Phys. Lett. 267B (1991) 366; Kaluza-Klein origin for the superstring tension, Phys. Rev. D45 (1992) R3321.

[14] E. Bergshoeff and P.K. Townsend, Super D-branes revisited, Nucl. Phys. B 531 (1998) 226;

E. Bergshoeff, D. Sorokin and P.K. Townsend, The M5brane Hamiltonian, Nucl. Phys. B533 (1998) 303.

[15] A. Achúcarro, J.P. Gauntlett, K. Itoh and P.K. Townsend, Worldvolume supersymmetry from spacetime supersymmetry of the four dimensional supermembrane, Nucl. Phys. B314 (1989) 129.

[16] K. Becker, M. Becker and A. Strominger, Fivebranes, membranes and non-perturbative string theory, Nucl. Phys. B456 (1995) 130.

[17] E. Bergshoeff, R. Kallosh, T. Ortín and G. Papadopoulos, Kappa-symmetry, supersymmetry and intersecting branes, Nucl. Phys. B502 (1997) 149.

[18] G.D. Robertson, Self-dual lumps and octonions, Phys. Lett. 249B (1990) 216.

[19] J.A. Harvey and A. Strominger, The heterotic string is a soliton, Nucl. Phys. B449 (1995) 535;

P.K. Townsend, String-membrane duality in seven dimensions, Phys. Lett. 354B (1995) 247;

S. Cherkis and J.H. Schwarz, Wrapping the M Theory fivebrane on $K_{3}$, Phys. Lett. 403B (1997) 225.

[20] S. Stanciu, D-branes in Kazama-Suzuki models, Nucl. Phys. B526 (1998) 295.

[21] C. Chryssomalakos, J.A, de Azcárraga, J.M. Izquierdo and J.C. Pérez-Bueno, The geometry of branes and extended superspaces, hep-th/9904137. 